\title{
Varieties of Misrepresentation and Homomorphism
}

\author{
Francesca Pero* $\quad$ Mauricio Suárez ${ }^{\dagger}$
}

\begin{abstract}
This paper is a critical response to Andreas Bartels' (2006) sophisticated defense of a structural account of scientific representation. We show that, contrary to Bartels' claim, homomorphism fails to account for the phenomenon of misrepresentation. Bartels claims that homomorphism is adequate in two respects. First, it is conceptually adequate, in the sense that it shows how representation differs from misrepresentation and non-representation. Second, if properly weakened, homomorphism is formally adequate to accommodate misrepresentation. We question both claims. First, we show that homomorphism is not the right condition to distinguish representation from misrepresentation and non-representation: a "representational mechanism" actually does all the work, and it is independent of homomorphism - as of any structural condition. Second, we test the claim of formal adequacy against three typical kinds of inaccurate representation in science which, by reference to a discussion of the notorious billiard ball model, we define as abstraction, pretence, and simulation. We first point out that Bartels equivocates between homomorphism and the stronger condition of epimorphism, and that the weakened form of homomorphism that Bartels puts forward is not a morphism at all. After providing a formal setting for abstraction, pretence and simulation, we show that for each morphism there is at least one form of inaccurate representation which is not accommodated. We conclude that Bartels' theory - while logically laying down the weakest structural requirements - is nonetheless formally inadequate in its own terms. This should shed serious doubts on the plausibility of any structural account of representation more generally.
\end{abstract}

\footnotetext{
*Department of Philosophy, University of Florence, Via Bolognese 52, 50139 - Firenze. E-mail address: francesca.pero@unifi.it

${ }^{\dagger}$ Department of Logic and Philosophy of Science, Faculty of Philosophy Complutense University of Madrid, 28040 - Madrid, Spain. Institute of Philosophy, School of Advanced Study, London University, Malet Street, WC1E 7HU - London, UK. E-mail address: msuarez@filos.ucm.es
} 


\section{Structural Approaches}

There is by now a long tradition of structural approaches to scientific representation, starting in van Fraassen (1980) and Suppes (1967) to the most sophisticated recent accounts by Pincock (2012) and Bartels (2006). The tradition's critics (Giere 1999, Frigg 2006, Suárez 2003, van Fraassen 2008, Contessa 2011) have invoked putative counterexamples to structural notions, displaying instances of scientific modeling where a model $\mathbb{B}$ is accepted as a representation of some object, system or process $\mathbb{A}$, while failing to hold the required structural morphism relation to $\mathbb{A}$. As a response, defenders of structural accounts have progressively weakened their constraints, from isomorphism to embedding, partial isomorphism and, most recently, to homomorphism. (Van Fraassen was both an early proponent, and nowadays a critic, at least in the terms defended here).

It is unclear in these papers what precise claims are being made on behalf of structural mapping or morphisms, and what exactly is the work that structures are supposed to perform. ${ }^{1}$ More worryingly, perhaps, the notion of structure itself remains imprecise and elusive. But whatever else is claimed on behalf of structural morphism, it is clear that the point of providing a structural account of representation is to provide some elucidation, however partial, of the central notion of scientific representation. Hence we shall take it that any structural account of representation is minimally committed to the claim that representation in science is a relation that is appropriately characterized or described as a kind of structural mapping or morphism. And indeed most authors in the tradition have invoked structural isomorphism and its variants as part of an analysis of representation. Thus for instance, it is claimed that:

$[\mathrm{T}] \mathrm{o}$ understand how an organism performs well using a certain representational system we have to consider the specific contents of the representation and how they relate to its reference objects. Content is a necessary component of representation, and homomorphisms are necessary to explain this necessary component. (Bartels 2006, 17)

The evidence for these claims and their reach remains nonetheless surprisingly unclear. It is in particular often unclear, as we shall point out in this article, whether isomorphism and its cousins are intended to provide an analysis of the notion of representation itself, or whether they are merely intended to describe some of the ways in which representation in science achieves some of its characteristic ends, such as for instance, the aim of accuracy. In other words, it is unclear whether structural mappings or morphisms are constitutive of representation in science, or merely some efficient means for representation to achieve its ends. Defenders of the structural accounts are often imprecise in shifting from evidence for the weaker case to claims in favor of the stronger constitutive claim. But the inference from

\footnotetext{
${ }^{1}$ We are using the term morphism to refer to any structural mapping regardless of the kind of transfer of structure from $\mathbb{A}$ to $\mathbb{B}$ that it implies. Therefore the term should not be understood as a synonym for homomorphism, which is at best the basic, or most elementary, form a morphism can take.
} 
the former to the latter claim is invalid, since the problem of representation and the problem of accurate representation are by now well-known to be distinct (Callender and Cohen 2006, Contessa 2007, Frigg 2006). We believe that there is so far no good argument to the effect that the evidence for the weaker claim (that structural morphisms are typically involved in the assessment of the accuracy of many mathematical representations in science) is also evidence for the stronger claim (that structural morphisms are constitutive of the nature of scientific representation, i.e. that a structural account of representation is correct). There are powerful independent arguments against the stronger claim (see Frigg 2006, Suárez 2003) that recommend a skeptical attitude to structural accounts of scientific representation in general.

In this paper we analyze the most sophisticated and plausible structuralist account of representation to date, namely Andreas Bartels' (2006) homomorphism account. The account's main virtue is the alleged capacity of homomorphism to account for the phenomena of misrepresentation, and indeed we believe this to be one of the greatest stumbling blocks for structural accounts. Hence we begin in Section 2 by reviewing the problem of misrepresentation in scientific modelling, in both the mistargetting and inaccuracy varieties. As an illustration of the latter, we briefly discuss the essential features of an elementary yet influential historical case of scientific modeling: the billiard ball model of gases. We argue that there are three ways in which scientific models typically misrepresent, and we refer to them as abstraction, pretence and simulation. We provide bare structural characterizations for all of them in terms of simple structural renditions of their representational sources and targets. We argue on the basis of the billiard ball model that scientific models abstract, many pretend, and some simulate; but that this does not take away any of their descriptive, predictive and explanatory value. Then in Section 3 we summarize Bartels' homomorphism theory of representation and review his claim that this theory accounts for misrepresentation. We point out the essential role adjudicated by Bartels to what he calls the "representational mechanism'. Representational mechanisms have a crucial role for representation (and misrepresentation) to occur and, since these mechanisms are independent of any structural mapping, we argue that misrepresentation is not accounted fully in structural terms. This particularly holds for mistargetting as presented in (Suárez 2003) In Section 4 we dispute the claim that misrepresentation as inaccuracy is accommodated within Bartels' structural account. We first point out that what Bartels calls homomorphism is in fact a stronger notion, namely epimorphism. We then show that epimorphism can not account for either abstraction, pretence or simulation. Turning to homomorphism proper, which is an extremely weak structural constraint, amounting to the relation technically known as completeness, we show that it can accommodate pretence kinds of misrepresentation, but not abstraction. Since we argue that most if not all scientific models abstract, it follows that even the weakest notion of structural morphism is too strong for scientific representation. The formal result is summarized in a table in the final section. In the concluding section, we admonish philosophers to take greater care with structural accounts of representation - while structural morphisms may provide good and valuable resources to 
assess the accuracy of many mathematical models in science, they can not actually account for the very relation of representation.

\section{Misrepresentation: Mistargetting and Inaccuracy}

"Scientific representations misrepresent": This is one of the main points of agreement in the recent literature on scientific representation. Any philosophical theory or account of scientific representation must not only accommodate but also explain minimally how representations fail to accurately characterize or describe their entire subject. Representations always simplify to some degree: this is at the heart of why they are useful in practice. ${ }^{2}$ Thus it would be a major objection to any philosophical account of representation that it does not account for misrepresentation. This has often been an issue for structural accounts - since on such accounts the conditions for the accuracy of a representation (the 'matching' of relations and properties at the source and target end) are also the very conditions for establishing the relationship of representation in the first place. This is most evident an objection to isomorphism accounts, and the relevant question for us is the extent to which the objection can be answered by means of suitable weakenings of the isomorphism relation. Yet, there is a stronger form that the objection may take, which we would like to consider in this section.

The stronger objection begins with the observation that there are distinct forms of misrepresentation and that these pose significantly different challenges for structural accounts. Two main kinds were already identified in Suárez (2003) and referred to there as mistargetting and inaccuracy. A model may misrepresent by being applied to the wrong target, perhaps as a result of having been mistakenly taken to be a different model in some particular context. The model's target is selected as part of the normative practice of model building that gives rise to it, but a particular agent may, perhaps out of lack of information or competence, apply it to the wrong target. The model is in that very context misrepresenting in a rather strong sense: it is used as a representation of a system or object that it is not intended for. We return to the issue later on in addressing whether Bartels' account actually provides necessary and sufficient conditions for representation, and whether these conditions can in some sense be thought to be 'structural'. For now, we focus only on the varieties of inaccurate misrepresentation. More specifically we discuss three forms that inaccuracy can take, and which we refer to as abstraction, pretence, and simulation.

The rough and ready definition of these terms is as follows: An abstraction essentially neglects some of the features of the target system it is about; a pretence ascribes to the target system features that this does not possess; a simulation both abstracts and pretends: it both neglects some of the actual features of the system and ascribes features to the system that it does not possess. We discuss these distinctions in relation to one of the best known and most widely discussed examples of an analogical model in the history of philosophy of

\footnotetext{
${ }^{2}$ Jorge Luis Borges' wonderful discussion of the one-to-one scale map is an exemplary parody of how a perfectly accurate representation is also perfectly useless (Borges 1954).
} 
science, namely the so-called 'billiard ball model' (Hesse 1970). Hesse presents this model as consisting of a negative, positive and neutral analogy between macroscopic billiard balls and gas molecules in a container. Thus in her famous dialogue between the Duhemist and the Campbellian, the Campbellian lists the properties of billiard balls and classifies them in three groups in relation with the analogy with gas molecules. In the negative analogy (the properties that pertain to billiard balls but not gas molecules) there are colour, hardness, brightness; in the positive analogy (the properties that billiard balls and gas molecules share) there are motion and impact. But there is a third group of properties that constitute what Hesse calls the neutral analogy. These are in the words of Hesse's Campbellian, "the properties of the model about which we do not yet know whether they are positive or negative analogies: These are the interesting properties, because [...] they allow us to make new predictions." (Hesse 1970, 8).

Now, Hesse does not describe them, but there is a further group of properties of interest in the analogical relationship between billiard balls and gases; these are the properties of the gas that are most definitely not properties of billiard balls. For instance, the billiard ball model captures microscopic features of elastic collisions between gas molecules to some extent, but it does not say anything informative regarding the macroscopic features of the gas, such as volume, density and pressure. We find ways to draw inferences to those macroscopic properties from the fully developed kinematical theory of gases, but there are no correlates in a system of billiard balls for such properties. What's more, the billiard ball model is positively misleading as a guide for such properties, since there is no relation in a system of billiard balls between average speed of the balls and the pressure exerted outwards by the system. Obviously the missing ingredient is free expansion, which is a thermodynamically irreversible property of any system of gas molecules, but has no equivalent or corresponding property in any dynamical feature of elastic collisions between classical particles or massive bodies, such as billiard balls. We could call this the 'inverse negative analogy' (or negative analogy 'by denial'): they are the properties that pertain to gas molecules but not billiard balls. They may even be explicitly denied for billiard balls (as indeed is the case with free expansion).

In fact, as some careful reading will reveal, the inverse negative analogy is of particular relevance in Campbell's original discussion of the example (see Campbell 1957). And there is some sense to this. Hesse had her own reasons to suppress the discussion of the inverse negative analogy which could only take away from the neutral analogy which she deemed fundamental. It is well known that her chief aim was to defend the thesis that the neutral analogy was key to the heuristics of research, and fully informed its logic. Campbell, however, was mainly preocuppied with the relation between theory and measurement, and more particularly with the theoretical presuppositions underlying measurement procedures. In this context the inverse negative analogy is relevant, for the macroscopic thermodynamic properties in question are measurable in the laboratory, while the internal microscopic properties of the gas can only by hypothesized or inferred from observation via the model.

There are further interesting differences between Hesse's discussion of Campbell's ex- 
ample and Campbell's original discussion. Perhaps the most striking is that Campbell never actually employs the term "billiard ball model". In fact, he does not refer to billiard balls once! His analogy is more generally with a system of perfectly elastic macroscopic balls - and, of course, billard balls are an approximate instance of these, even though they are not in reality perfectly elastic. But the analogy is fit for most relevant purposes, since it captures some essential aspects of the relationship between the laws that apply to both gas molecules and macroscopic yet point-size elastic balls. As Campbell writes: "The propositions of the hypothesis of the dynamical theory of gases display an analogy [...] to the laws which would describe the motion of a large number of infinitely small and highly elastic bodies contained in a cubical box." (1957, 128). There are however some important points of difference where the model most definitely goes astray, and they can not be understood to be part of Hesse's negative analogy, since they comprise properties of the gas molecules that the model fails to describe correctly altogether. These properties, which comprise what we refer to as the inverse negative analogy, include free expansion, but also thermal conductivity, and viscosity. As Campbell puts it: "The relation predicted [between pressure, density, and temperature of the gas and its viscosity] does not accord with that determined experimentally; in particular it is found that the theory predicts that the coefficient of viscosity will be be determined by the size and shape of the containing vessel, whereas experiment shows that it depends, in a given gas, only on the density and temperature." (ibid., 134).

While there is no space here to discuss the details fully, the considerations above already suggest the following distinctions with respect to the ways in which the elastic macroscopic balls model misrepresents gases. First of all, there are all the properties of the model elements which are missing in the gases: they constitute the negative analogy in Hesse's terms. Thus billiard balls are shiny and hard, but gas molecules are not (they are neither hard nor soft; neither shiny nor opaque). We may then say that the model pretends with respect to its target system. It may seem easy to discharge these properties by simply redefining the model to include only the positive and neutral analogies. Thus, one may insist, the analogy is not meant with billiard balls per se but with constructs that are like billiard balls except in those respects in which billiard balls are positively unlike gas molecules. But there are a number of problems with this strategy, some of which were already discussed by Hesse. For a start, the move is of course circular as a definition of the function of the analogy - since it requires us to already have a hang on what properties are and are not actually analogous. And things get even worse when we notice that there are also properties of gases that the system of elastic balls - whether or not billiard balls - can not possibly be said to have, including thermal conductivity, viscosity and free expansion. This is the inverse negative analogy we are emphasizing here and we may say that the model abstracts in this case. The analogy as based upon the model denies that the gas has these properties. In some cases the model even positively misleads regarding the character of such properties in the gas. If we consider viscosity in the example above, we see that the fact that the model fails to describe it correctly depends on the fact that it abstracts 
from density and temperature on which viscosity actually depends. Instead, according to the model, viscosity rather depends on properties the billiard ball model pretends about, such as the size and shape of the containing vessel. In these cases we concurrently abstract and pretend about a property of the target system, thus simulating it. We then say that a model lies about its target whenever it is deceptive in this sense about it. There is no sense in which this can be put down to mere "heuristics". Rather, as Campbell insists, the model analogy is not to be considered a mere heuristics in the development of a new theory, but must be understood to be part of the theory itself: "It is often suggested that the analogy leads to the formulation of the theory, but that once the theory is formulated the analogy has served its purpose and may be removed and forgotten. Such a suggestion is absolutely false and perniciously misleading" $(1957,129)$.

Thus we must take seriously that models misrepresent by abstracting away, and thus ignoring, certain properties of the target system (escape velocity), by pretending that certain properties of the target system do obtain which actually do not (hardness and shine) and by simulating, that is, by misleadingly denying that some properties obtain which in fact do (viscosity, thermal conductivity). What's more for some and the very same elements, a model will typically both abstract with respect to some property, and pretend with respect to some other. In other words, the representation by models will typically involve both ignoring certain properties that do obtain and postulating other properties that do not obtain even for the very same sets of elements in the domain of the model.

Now, let us attempt to represent these distinctions somewhat more formally, in what we regard as a hospitable framework for structuralism, which assumes that there are uncontroversial structural representations of both source and target. This is a strong assumption, but without which the structuralist conception of representation does not even get off the ground. ${ }^{3}$ Thus consider a model and its target as two relational structures, $\mathbb{B}=\left\langle B,\left(R^{B}\right)\right\rangle$ and $\mathbb{A}=\left\langle A,\left(R^{A}\right)\right\rangle$, with their own domains of individuals, $A$ and $B$, and the sets of relations defined over the domains: respectively $\left(R^{A}\right)$ and $\left(R^{B}\right)$. $\mathbb{A}$ and $\mathbb{B}$ are assumed to be similar structures: while the elements of $A$ and $B$ may be different, the corresponding relations in $R^{A}$ and in $R^{B}$ have the same number of arguments (Dunn and Hardegree 2001,

\footnotetext{
${ }^{3}$ One of the referees points out that this is in fact an incredibly strong assumption. As he or she puts it: "A system of gas molecules is not a set of elements and a family of labelled relations, etc. It has no labelled relations because it contains no labels [...] The real world thing being represented is not a structure, whereas the author's 'target' has to be a structure for the author's discussion to make any sense at all". We agree wholeheartedly with this referee. It is indeed the case that a real physical object, a system, or a phenomenon, can only be said to be a structure under a description. And it is clear (as one of us has often pointed out, in e.g. Suárez (2010, p.96)) that any structural description is necessarily vastly underdetermined: Every real object exemplifies multiple, perhaps an infinite number of, structures. This simple fact puts great pressure upon structuralist claims regarding ontology (to the extent that claims to the effect that the "world consists only of structure" or some such thing, are rendered vacuous or, worse, incoherent - as pointed out by e.g. van Fraassen (2006).) We ignore this issue because almost all the literature that we do address ignores it too, and also because it can only strengthen our critique of the homomorphism theory of representation. But it is worth pointing out with the referee that general widespread acquiescence with a false assumption does not make it any less false or unwarranted.
} 
10).

We use the bar symbol for tuples of elements of $A$ and $B: \bar{a}=\left(a_{1}, \ldots, a_{n}\right) \in A^{n}$ and $\bar{b}=\left(b_{1}, \ldots, b_{n}\right) \in B^{n}$.

We say that a model $\mathbb{B}$ abstracts some property $R_{j}^{A} \subseteq A^{n}, j \in\{1, \ldots, m\}$, of a target system $\mathbb{A}$ if and only if there exists $\bar{a} \in A^{n}$ such that $R_{j}^{A}(\bar{a}) \wedge \neg R_{j}^{B}(f(\bar{a}))$, where $R_{j}^{B} \subseteq B^{n}$ is the corresponding relation of $R_{j}^{A}$ in $\mathbb{B}$ and $f$ is a mapping from $A$ to $B$. The abstracted properties are in the inverse negative analogy, or negative analogy by denial. We then say that the model $\mathbb{B}$ pretends some property $R_{k}^{B} \in B^{n}, k \in\{1, \ldots, m\}$, of the target system $\mathbb{A}$ if and only if there exists $\bar{b} \in B^{n}$ and $\bar{a} \in A^{n}$ such that $\bar{b}=f(\bar{a})$ and $\neg R_{k}^{A}(\bar{a}) \wedge R_{k}^{B}(f(\bar{a}))$, where $R_{k}^{A} \subseteq A^{n}$ is the corresponding relation of $R_{k}^{B}$ in $\mathbb{A}$. The pretended properties are typically in the negative analogy as originally discussed by Hesse. Finally, we say that a model $\mathbb{B}$ simulates a target $\mathbb{A}$ when it both abstracts and pretends some properties of the same elements of $A$ and of their images in $B$; formally, for some tuple $\bar{a} \in A^{n}$ with $\bar{b}=f(\bar{a}) \in B^{n}$, some $R_{j}^{A}, R_{k}^{A} \subseteq A^{n}$ and $R_{j}^{B}, R_{k}^{B} \subseteq B^{n}$, it is true that $R_{j}^{A}(\bar{a}) \wedge \neg R_{j}^{B}(f(\bar{a}))$ and $\neg R_{k}^{A}(\bar{a}) \wedge R_{k}^{B}(f(\bar{a}))$.

We have argued in this section, by appeal to a well-known foundational example in the literature, that models typically simulate their targets, by both abstracting some of their properties away and misleadingly asserting some of the properties they do not actually possess. We next turn to the best candidate we know for a structuralist conception of representation, namely Bartels' homomorphism theory, and argue that it can not accommodate these features.

\section{Bartels' Homomorphism Theory and the 'Representational Mechanism'}

The main tenets of a structural account of scientific representation can be summarized as follows: (i) model sources and their targets exemplify, instantiate, possess or at any rate may be described as relational structures in the sense of mathematical logic, or set-theory; (ii) a model represents a target system only if the relations in the target are partially or totally transferred to the model via some sort of morphism.

We have provided a definition for relational structure in the previous section. The transfer required by condition (ii) is accomplished by some function $f: A \rightarrow B$. In model theory a twofold role is ascribed to $f$. As a mapping, $f$ assures that each individual in $A$ has one, and only one, corresponding element (an image) in B. But in addition, as a morphism, $f$ is a structure preserving mapping and it assures that related objects possess related properties. The existence of a morphism between the model and its target is what the advocates of the structural approach take to be the condition for representation: $a$ model $\mathbb{B}$ represents a target system $\mathbb{A}$ (if and) only if $\mathbb{B}$ is to some extent morphic to $\mathbb{A}$.

Isomorphism is sometimes advocated as the basic morphism between structures. For $f: A \rightarrow B$ to be an isomorphism, several conditions need to be met. First, $f: A \rightarrow B$ 
must be a bijective function, that is, for every $b \in B$ there exists an $a \in A$ such that $f(a)=b$ (also known as surjectivity) and, for every $a, a^{\prime} \in A$, if $a \neq a^{\prime}$ then $f(a) \neq$ $f\left(a^{\prime}\right)$ (injectivity). Second, for all $j$ and all elements $a_{i}$ of $A: R_{j}^{A}\left(a_{1}, \ldots, a_{n}\right)$ if and only if $R_{j}^{B}\left(f\left(a_{1}\right), \ldots, f\left(a_{n}\right)\right)$. In other words, all relations in $\mathbb{A}$ are transferred to $\mathbb{B}$ so that the two structures are relationally identical, in the sense that the properties they define have identical features. (The structures themselves are obviously not identical since their domains contain different elements).

The idea that isomorphism may constitute representation has been criticized on several grounds. There are first of all urgent questions regarding the fundamental assumption that model sources and targets are or may be said to possess structures. For instance, van Fraassen (2008) suggests that isomorphism alone cannot serve as a condition of representation because, he argues, the structure $\mathbb{A}$ is a "relevant mathematical representation" (ibid, 243) of the target system to be represented only by construction. That is, we must first of all choose a domain of elements $A$ and a set $R^{A}$ of relations for it as a description of the target system or phenomenon. The claim that a model $\mathbb{B}$ is isomorphic to $\mathbb{A}$, which allows to use $\mathbb{B}$ as a representation of $\mathbb{A}$, depends on the prior act of construction of $\mathbb{A}$ which is essentially a conventional and pragmatic choice.

Another class of objections, raised by Suárez (2003) and reiterated by Frigg (2006), undermine the attempt to reduce representation to the relation of isomorphism, irrespective of whether the fundamental assumption that model sources and targets are structures or may be described as such. Thus the logical argument shows that isomorphism and representation do not share logical properties: while isomorphism is reflexive, symmetric and transitive, representation is non-reflexive, non-symmetric and non-transitive. The nonsufficiency and non-necessity arguments show that representation may fail to obtain when isomorphism holds (non-sufficiency), and may obtain when isomorphism does not (nonnecessity). Finally the misrepresentation argument appeals to the already mentioned fact that inaccuracy is intrinsic to all scientific representation, while isomorphism seems to leave no room for either incomplete or incorrect representation.

In response to these objections the advocates of the structuralist account have proposed weakenings of the isomorphism relation. For instance, Andreas Bartels (2006) suggests that homomorphism will serve to overcome at least the misrepresentation objection. Roughly speaking, what allegedly makes homomorphism immune to the criticisms undermining isomorphism is the fact that homomorphism allows some parts of the model not to have any counterparts in the target, thus leaving the necessary room to account for inaccurate representation.

Bartels explicitly endorses the structural account of representation when he claims that homomorphism is a necessary condition for representation: "something, $\mathbb{B}$, can represent something, $\mathbb{A}$, only if some structure of the represented domain $\mathbb{A}$ is transferred to its image $\mathbb{B}$ " (ibid., 7) and that: " $\mathbb{B}$ represents $\mathbb{A}$ only if $\mathbb{B}$ is a homomorphic image of $\mathbb{A}$ " (ibid., 8). The homomorphism account of representation advocated by Bartels in fact comprises two parts. One part is purely formal, and treats homomorphism model-theoretically. The other 
part concerns the application of the concept 'being homomorphic to' and claims that this concept is extensionally equivalent to 'to potentially represent'. Both the formal and the extensional analyses of homomorphism provided by Bartels play a role in his attempt to show that homomorphism accounts for misrepresentation, so let us look at them in turn.

According to Bartels' definitions, the following three conditions must obtain for a structure $\mathbb{B}$ to be homomorphic to $\mathbb{A}$ : for all $j$, all $\left(a_{1}, \ldots, a_{n}\right)$ in $A^{n}$, and all $\left(f\left(a_{1}\right), \ldots, f\left(a_{n}\right)\right)$ in $B^{n}$ :

$$
\begin{array}{cr}
\text { Completeness: } & \text { if } R_{j}^{A}\left(a_{1}, \ldots, a_{n}\right) \text {, then } R_{j}^{B}\left(f\left(a_{1}\right), \ldots, f\left(a_{n}\right)\right) \\
\text { Faithfulness: } & \text { if } R_{j}^{B}\left(f\left(a_{1}\right), \ldots, f\left(a_{n}\right)\right) \text { then } R_{j}^{A}\left(a_{1}, \ldots, a_{n}\right) \\
\text { Surjectivity: } & \text { for every } b \in B, \text { there exists } a \in A \text { such that } f(a)=b
\end{array}
$$

The condition of surjectivity on $f$ assures that all the elements in $B$ are images of one or more element in $A$. Completeness rules out the possibility that there is a relation in $\mathbb{A}$ which has not a counterpart in $\mathbb{B}$, so that the information that $\mathbb{B}$ provides about $\mathbb{A}$ is complete. On the other hand, faithfulness rules out that there is a relation in $\mathbb{B}$ which has not a counterpart in $\mathbb{A}$, so that $\mathbb{B}$ provides a faithful snapshot of the relational framework in $\mathbb{A}$. We then say that $\mathbb{B}$ is homomorphic to $\mathbb{A}$.

The relation of homomorphism thus defined identifies the set or class of structures to which any structure $\mathbb{B}$ is homomorphic, what we may call its homomorphism class. According to Bartels, these structures constitute the representational content of $\mathbb{B}$, that is, they are all potential representational targets of $\mathbb{B}$. In order for any of these potential targets to turn into the actual target of $\mathbb{B}$, a representational mechanism must pick it out from the homomorphism class as the target for $\mathbb{B}$. A representational mechanism can be of two kinds: it may arise from an agent's intentions and purposes (an intentional representational mechanism), or it may be the result of naturally occurring causal relations (a causal representational mechanism). In the first case, the selection of the actual target from the homomorphism class is arbitrary, depending entirely on an agent's purposes, while in the second case the selection is driven by some causal facts that are independent of the agent. In either case, the representational mechanism has in effect the absolutely ineliminable role of picking out the actual representational target of a particular model $\mathbb{B}$. In spite of this, Bartels claims that his theory retains its structural character, since homomorphism is nonetheless "the necessary condition of correct actual representation" (ibid., 12). Let us inspect this claim a little closer.

Two forms of misrepresentation are generally considered in the literature: inaccuracy and mistargetting. ${ }^{4}$ The three kinds of misrepresentation presented in Section 1 all lead to inaccuracy, which is misrepresentation in the broad sense. As for mistargetting, it is "the phenomenon of mistaking the target of a representation" (Suárez 2003, 233).

Now, homomorphism theory is claimed by Bartels to be conceptually adequate, that is, it sharply distinguishes cases where $\mathbb{B}$ represents, $\mathbb{B}$ does not represent, and $\mathbb{B}$ misrepresents.

\footnotetext{
${ }^{4}$ While misrepresentation as inaccuracy is taken into account in Cartwright (1983), Giere (1988), Teller (2001, 2008), Suárez (2003, 2004), Frigg (2006), van Fraassen (2008), Pincock (2011), Contessa (2011), misrepresentation as mistargetting is presented in Suárez $(2003,2004)$.
} 
This claim is relevant insofar conceptual inadequacy is the usual grounds against structural accounts in the literature. Indeed, it is argued, the fact that structural accounts treat morphisms as necessary conditions for representation leaves no room for the intermediate condition 'there is representation and it is incorrect': either there exists a morphism from $\mathbb{A}$ to $\mathbb{B}$, hence representation, or there exist not morphism and representation does not obtain.

According to Bartels, such a charge would be unfair to his homomorphism theory. Indeed, the distinction between the representational content of $\mathbb{B}$ and its target allows the theory to account for the following situation:

If a reference object for $\mathrm{B}[\mathbb{B}]$ is chosen by a representational mechanism out of the set of objects potentially represented by $B[\mathbb{B}]$, then $B[\mathbb{B}]$ will correctly represent this object. If a reference object for $B[\mathbb{B}]$ is chosen which does not belong to this set, then this reference object will be misrepresented by $\mathrm{B}[\mathbb{B}]$. Thus, the case in which something $A[\mathbb{A}]$ is misrepresented by $B[\mathbb{B}]$ and the case in which $A[\mathbb{A}]$ is not represented by $B[\mathbb{B}]$ (i.e. $A[\mathbb{A}]$ is not a reference object of $\mathrm{B}[\mathbb{B}])$ are clearly distinct. $(2006,14)$

The distinction between target and content of $\mathbb{B}$ plays then a crucial role in accommodating those intermediate cases where representation occurs, and it is not correct. In order to illustrate misrepresentation thus conceived, let's consider a universe of discourse which allows the following five structures $\left\{\mathbb{B}, \mathbb{A}_{1}, \mathbb{A}_{2}, \mathbb{A}_{3}, \mathbb{A}_{4}\right\}$. Among the five structures, only $\mathbb{A}_{1}$ and $\mathbb{A}_{2}$ are homomorphic to $\mathbb{B}$. We call $\mathbb{H}$ the set containing $\mathbb{A}_{1}$ and $\mathbb{A}_{2}$, which then constitute the representational content of $\mathbb{B}$. Now suppose that a representational mechanism picks $\mathbb{A}_{3}$ as the target of $\mathbb{B}$, thus misrepresenting $\mathbb{A}_{3}$. Consequently, structure $\mathbb{A}_{4}$ is neither a potential target of $\mathbb{B}$, nor misrepresented by $\mathbb{B}$. Providing a sharp distinction between representing (picking a target within $\mathbb{H}$ ), non-representing (having a structure neither belonging to $\mathbb{H}$ nor picked by a representational mechanism), and misrepresenting (having a structure not belonging to $\mathbb{H}$ and nonetheless picked as a target), the homomorphism theory has the resources to satisfy conceptual adequacy, thus explaining misrepresentation. In particular, this notion of misrepresentation may be seen to be addressing directly the concerns raised by Suárez (2003) about mistargetting: the act of ascribing a target outside the representational content of $\mathbb{B}$ may be thought to make his notion of mistargetting precise.

However, Bartels' homomorphism is only allegedly conceptually adequate. Bartels claims that we have misrepresentation if a representational mechanism picks a target for $\mathbb{B}$ outside the set $\mathbb{H}$ of all the structures $\mathbb{B}$ is homomorphic to. Misrepresentation is then the act performed by a representational mechanism to choose as a target for $\mathbb{B}$ a structure which $\mathbb{B}$ is not homomorphic to. Of course, homomorphism is necessary to identify the set $\mathbb{H}$ of structures over which neither non-representation nor misrepresentation can occur. However, before a representational mechanism choses a target for $\mathbb{B}$ among the structures outside $\mathbb{H}$, any of these structures could be either misrepresented or non-represented at all. Therefore, it is the choice made by a representational mechanism to actually determine 
which structure is misrepresented and, consequently, which one is not represented. In other words, homomorphism alone can not help in sharply distinguishing representation, nonrepresentation and misrepresentation. This is because misrepresentation is after all a case of representation - just an incorrect one. Homomorphism was never supposed for Bartels to be a sufficient condition for representation (or mis-representation). But what his discussion of the representational mechanism reveals at this point is that he does not actually take it to be necessary either - since the representational mechanism on its own, on Bartels' admission, is able to select a representational target, whether it be one outside or inside the homomorphism class. (Otherwise we could not even express the thought that the structure $\mathbb{A}_{3}$ outside the homomorphism class is mis-represented.) It follows that being picked out by the representational mechanism is what is really necessary, regardless of homomorphism. ${ }^{5}$ Nonetheless, the non-sufficiency of homomorphism undermines the conceptual adequacy invoked for the theory built on it. That "a representational intention has to occur" (p. 12 ) in order to finalize the distinction between the non-represented and the misrepresented contravenes the claim that homomorphism "permits" misrepresentation. As Bartels claims, the reason why homomorphism could be claimed to fail conceptual adequacy is that "a homomorphism between relational structures $[\mathbb{A}]$ and $[\mathbb{B}]$ either exists or does not exist; in the first case, $[\mathbb{B}]$ represents $[\mathbb{A}]$, whereas in the second case $[\mathbb{B}]$ does not represent $[\mathbb{A}]$ ", thus leaving unexplained "What would it mean for $[\mathbb{B}]$ to represent $[\mathbb{A}]$, but incorrectly?". We have shown that Bartels' theory does not break this deadlock: if there is homomorphism, there is representation; if there is not, we are left with an indistinct whole where it is not clear what is not represented and what is misrepresented.

Bartel's homomorphism theory falls short also as an account of misrepresentation as mistargetting. For the original objection raised by Suárez was not reliant on the possibility of ascribing a target that lies outside of the homomorphism class. To pursue the example above, the objection does not trade on the actual representational target $\mathbb{A}_{3}$ lying outside the homomorphism class at all. The objection can be entirely run within the homomorphism class, and in fact it properly belongs there. For Suárez's point is that the mistaken target is assumed wrongly to be the target precisely because it holds the required structural relation, and merely on account of this fact. The point of misrepresentation by mistargetting is rather that no structural characterization can distinguish structures within the homomorphism class regardless of whether they are or not picked out as the actual target. In other words, suppose that the representational mechanism above picked out $\mathbb{A}_{1}$ as the representational target of $\mathbb{B}$ and that someone mistakenly identifies $\mathbb{A}_{2}$ as the target for $\mathbb{B}$. Then there is no available structural characterization of this mistake since both structures are on equal terms in the homomorphism class of $\mathbb{B}$. It should be clear that this point survives Bartels'

\footnotetext{
${ }^{5}$ In fact, Bartels' attempt to accommodate the conceptual adequacy seems to resolve in a form of deflationary, or functional, account. Deflationary (Suárez 2004) or functional (Chakravartty 2010) approaches treat representation as a function of models which allows model users to gain information about the target at stake via the model. The ascription, or recognition, of the representational function of a model by a user is then essential to have representation. The crucial role played by the representational mechanism's choice in Bartels' homomorphism theory puts his theory very much in line with those accounts.
} 
disquisition in the quote above entirely.

Homomorphism theory seems then to fall short of what would be required for an adequate account of scientific representation even by Bartels' own standards. What we need to see now is whether the homomorphism theory fares any better in dealing with misrepresentation as inaccuracy, which is after all where the promise of the account lies in the first place.

\section{Structural Morphisms and Representational Inaccuracy}

We need to see now if the formal analysis fares any better than the extensional analysis and enables the homomorphism account to accommodate the inaccuracy kinds of misrepresentation. We have seen that Bartels identifies three conditions for homomorphism (sect. $3)$ : completeness, faithfulness and the condition that the $f: A \rightarrow B$ be surjective. These conditions, if weakened, might "fit the cases in which representations do not work perfectly" (Bartels 2006, 9). In such cases, Bartels argues, representation may either "lead to false expectations concerning facts in the represented domain" or "blur some of the fine grained differences existing in the represented domain" (ibid.). These are precisely cases of misrepresentation as inaccuracy. In particular, they do recall the formulation we put forward for, respectively, pretending and abstracting. This is why in what follows we treat Bartels' formal analysis of homomorphism as an attempt to accommodate misrepresentation as inaccuracy.

\subsection{Homomorphism versus Epimorphism}

Before proceeding, we need to point out a technical issue about the notion of homomorphism advocated by Bartels. In the literature, the only condition required for homomorphism is completeness, i.e., the condition which assures that every fact in $\mathbb{A}$ has a corresponding (atomic or relational) fact in $\mathbb{B} .{ }^{6}$ On the other hand, a surjective homomorphism is the condition for $\mathbb{B}$ to be the homomorphic image of $\mathbb{A} .{ }^{7}$ Therefore, the notion of homomorphism that Bartels is appealing to does not coincide with the standard notion of homomorphism nor with homomorphic image. Indeed, besides completeness and surjectivity of $f$, Bartels requires an additional condition, namely faithfulness:

If (i) [faithfulness] and (ii) [completeness] are fulfilled, $f$ is a homomorphism from $\mathbb{A}$ onto $\mathbb{B}$, and $\mathbb{B}$, by virtue of the existence of $f$, can be said to be an homomorphic image. (Bartels 2006, 8)

\footnotetext{
${ }^{6}$ See Chang and Keisler (1973), Dunn and Hardegree (2001), Hodges (1997), Hodges and Scanlon (2013)

7 "A relational structure $\mathbf{B}$ is said to be a homomorphic image of $\mathbf{A}$ if there exists a homomorphism from $\mathbf{A}$ to $\mathbf{B}$ that is onto $B$ (in symbols, $\mathbf{B}=h^{*}(\mathbf{A})$ ). (A function $f$ maps $\mathbf{A}$ onto $\mathbf{B}$ [it should be $A$ onto $B$ ] if for every $b \in B$ there is an $a \in A$ such that $h(a)=b$ )." (Dunn and Hardegree 2001, 15). Read the bold character in the quote as our $\mathbb{A}$ and $\mathbb{B}$.
} 
It should be noted, however, that a homomorphic image is not necessarily also a faithful one. Indeed, the structure $\mathbb{B}$ can be a homomorphic image of $\mathbb{A}$ and yet bear a relation $R_{j}^{B}$ which has no counterpart in $\mathbb{A}^{8}$

Our claim is that the morphism on which Bartels grounds his structural account is not really homomorphism. Indeed, Bartels claims for the kind of morphism employed in his theory more properties than those exhibited by standard homomorphism, i.e., surjectivity and faithfulness. A good candidate for the notion of homomorphism as employed by Bartels is the notion of epimorphism as presented by Rothmaler (2005, sect.2, 474), who claims for it the same properties as Bartels: surjectivity, faithfulness and completeness. ${ }^{9}$ Although Rothmaler's notion as well is not standard, we find it appealing for two reasons. First, it helps us to provide a reliable taxonomy of morphisms, which has been long overdue in the literature. Second, the taxonomy thus obtained is a useful device to evaluate the adequacy of those theories of representation built on morphisms, as shown in section 4.2.

We can now consider the weakenings which, according to Bartels, allow epimorphism to accommodate misrepresentation as inaccuracy. The first form of weakening is on faithfulness and it leads to the notion of minimal fidelity (Bartels 2006, 9). While faithfulness in its original formulation (2) requires that the implication $R_{j}^{B}(f(\bar{a})) \rightarrow R_{j}^{A}(\bar{a})$ holds for all the counterimages of $f(\bar{a}) \in B^{n}$, all $j, R_{j}^{A} \in A^{n}$, and $R_{j}^{B} \in B^{n}$, minimal fidelity allows the implication to hold for some of the counterimages only. In other words, minimal fidelity admits the following case:

$$
\text { there exists a tuple } \bar{b} \in B^{n} \text { and } \bar{a} \in A^{n}, f(\bar{a})=\bar{b}: R_{j}^{B}(f(\bar{a})) \wedge \neg R_{j}^{A}(\bar{a})
$$

The fact that epimorphism is not necessarily injective is crucial here since a one-to-one correspondence between the arguments in $A$ and their images in $B$ would make the conditions of faithfulness and minimal fidelity equivalent: given that each $b_{i} \in B$ in the range of $f$ has only one counterimage $a_{i} \in A^{n}$, it is just equivalent to claim that the conditional $R_{j}^{B}(\bar{b}=f(\bar{a})) \rightarrow R_{j}^{A}(\bar{a})$ holds for all the tuples of counterimages of $\bar{b}=f(\bar{a}) \in B^{n}$, or that it holds for at least one tuple.

\footnotetext{
${ }^{8}$ Consider two similar structures, $\mathbb{A}=\left\langle A,\left(R_{1}^{A}, R_{2}^{A}\right)\right\rangle$ and $\mathbb{A}=\left\langle B,\left(R_{1}^{B}, R_{2}^{B}\right)\right\rangle$, with $A \in \mathbb{A}=$ $\left\{a_{1}, a_{2}, a_{3}, a_{4}\right\}, B \in \mathbb{B}=\left\{b_{1}, b_{2}, b_{3}\right\}$. The mapping $f: A \rightarrow B$ is surjective, and the condition of completeness holds. Therefore, $\mathbb{B}$ is a homomorphic image of $\mathbb{A}$. To find a case where the conditions of completeness and the surjectivity of $f$ (and $\mathbb{A}$ and $\mathbb{B}$ are similar structures) are satisfied, but $\mathbb{B}$ is not faithful, we need a relation $R_{j}^{B} \in \mathbb{B}$ which has no counterpart $R_{j}^{A} \in \mathbb{A}$ and, at the same time, we need to assure that all the relations in $\mathbb{A}$ have their counterparts in $\mathbb{B}$. The function $f: A \rightarrow B$ is surjective (and not injective) and ascribes to each argument the following images: $f\left(a_{1}\right)=b_{1}, f\left(a_{2}\right)=b_{2}, f\left(a_{3}\right)=b_{3}, f\left(a_{4}\right)=b_{3}$. Consider now the case that $\mathbb{A}$ has the following family of relations: $R_{1}^{A} \subseteq A^{2}=\left\{\left(a_{1}, a_{2}\right),\left(a_{1}, a_{3}\right)\right\}$ and $R_{2}^{A} \subseteq A^{2}=\left\{\left(a_{1}, a_{2}\right),\left(a_{3}, a_{4}\right)\right\}$. As for $\mathbb{B}: R_{1}^{B} \subseteq B^{2}=\left\{\left(b_{1}, b_{2}\right),\left(b_{1}, b_{3}\right)\right\}$ and $R_{2}^{B} \subseteq B^{2}=\left\{\left(b_{2}, b_{1}\right),\left(b_{3}, b_{2}\right)\right\}$. The relation $R_{1}^{B}$ in $\mathbb{B}$ thus corresponds to both the relation $R_{1}^{A}$ and $R_{2}^{A}$ in $\mathbb{A}$, while the relation $R_{2}^{B}$ has no counterpart in $\mathbb{A}$. Therefore, $\mathbb{B}$ is a homomorphic image of $\mathbb{A}$ while faithfulness is violated.

${ }^{9}$ The standard definition of epimorphism is "surjective homomorphism". Therefore Rothmaler adds faithfulness as a further condition. As it will turn out in section 4.2, this notion of epimorphism works fine also to distinguish the conditions for having epimorphism from those required for having a "homomorphic image".
} 
The second form of weakening is on completeness, and it admits the case where some, or even all the relations in $\mathbb{A}$ are not preserved in $\mathbb{B}$. Weakening on completeness can take two forms: either some relations in $\mathbb{A}$ are not represented at all in $\mathbb{B}$, or some $n$-tuples in $\mathbb{A}$ are not represented at all in $\mathbb{B}$ (which is to say, some $n$-tuples of images in $\mathbb{B}$ do not stand in any relation of $\left\langle R^{B}\right\rangle$ although their counterimages stand in the corresponding relations $\left.\left\langle R^{A}\right\rangle\right)$.

It is worth noticing at this point the major difference between these two types of weakenings. The weakened form of faithfulness is a proper condition, in the sense that it does impose some restrictions on the transfer of structure: it cannot be the case that a relation in $\mathbb{A}$ does not have a corresponding relation representing it in $\mathbb{B}$. The weakened form of completeness, on the other hand, is not a condition at all, it rather consists in allowing any possible scenario, which is forecast by Bartels himself: "The fewer relations for which the transfer of structure holds, and the fewer the number of elements of A to which the transfer is restricted, the poorer the representation will be with respect to content. In an extreme case, no content will be left" (ibid., 11). Another, more astonishing, fact about weakened completeness is that it is a violation of the very minimal condition required for the transfer of structure (i.e. completeness) and, therefore, for there being a morphism at all. Indeed, to restrict the transfer of structure either to a certain range of arguments or to certain subsets of relations implies that $f$ is, respectively, neither a well-defined function nor a proper morphism. Thus no attempt to ground the representational relation on weakened completeness may be interpreted as providing a meaningful structural account of representation given that the very notion of morphism on which the account is claimed to be built would be left out. The relevant weakenings must be of a different kind. Let us see what Bartels proposes in order to accommodate inaccurate representations.

\subsection{Morphisms and Misrepresentation (as Inaccuracy)}

In the previous section we have introduced the weakenings which, according to Bartels, allow to accommodate misrepresentation as inaccuracy. In order to see whether they actually accomplish the task, here we confront each morphism, both in its standard and weakened version, with the formalized versions of abstraction, pretence and simulation that we introduced in Section 2. For the sake of completeness, our analysis will include also isomorphism which, as mentioned in the previous sections, is the morphism employed in other structural accounts. Isomorphism demands the following conditions to be satisfied: completeness, faithfulness, and that the mapping $f: A \rightarrow B$ be both injective and surjective. ${ }^{10}$ Our goal is then to verify that for every morphism there exists at least one form of misrepresentation which is not accommodated, thus showing that none of the three morphisms account for misrepresentation as inaccuracy. For the sake of clarity, we recapitulate in table (1) the conditions for each morphism, marking with a star the weakened morphisms that we have

\footnotetext{
${ }^{10}$ Dunn and Hardegree $(2001,17)$ consider the injectivity and surjectivity of $f$ only as a condition for isomorphism. Chang and Keisler (1973, 21), Hodges (1997, 5) and Robinson (1963, 25) consider also faithfulness as a condition for isomorphism.
} 


\begin{tabular}{|c|c|}
\hline Morphism & Characteristic Conditions \\
\hline Homomorphism & completeness \\
\hline Epimorphism & surjectivity of $f$, completeness, faithfulness \\
\hline Epimorphism $* c$ & surjectivity of $f$, weak completeness, faithfulness \\
\hline Epimorphism $* f$ & surjectivity of $f$, completeness, weak faithfulness \\
\hline Isomorphism & surjectivity and injectivity of $f$, completeness, faithfulness \\
\hline Isomorphism $^{* c}$ & surjectivity and injectivity of $f$, weak completeness, faithfulness \\
\hline Isomorphism*f & surjectivity and injectivity of $f$, completeness, weak faithfulness \\
\hline Epimorphism $* c, f$ & surjectivity of $f$, weak completeness, weak faithfulness \\
\hline Isomorphism $^{* c, f}$ & $\begin{array}{l}\text { surjectivity and injectivity of } f \text {, weak completeness, weak faith- } \\
\text { fulness }\end{array}$ \\
\hline
\end{tabular}

Table 1: Morphisms

discussed.

Two things need to be noted before proceeding. First, cases where the morphisms are weakened on completeness are not to be considered since, for the reasons presented in the previous section, they are not morphisms at all. Second, in table (1) the following two cases are not listed: the case of a surjective homomorphism, and the case of a faithful homomorphism (without surjectivity). The first case satisfies the conditions for $\mathbb{B}$ to be a homomorphic image of $\mathbb{A}$. The surjectivity of $f: A \rightarrow B$, however, is neither a sufficient nor a necessary condition for homomorphism, so it can be omitted for the sake of argument without any loss of generality. On the other hand, a faithful homomorphism $f$ which is not surjective is not an interesting case to consider, since faithfulness holds for the elements in $\mathbb{B}$ which are in the range of $f: A \rightarrow B$ only. And so in what follows, these quantifiers will be omitted whenever redundant.

We consider abstraction first, which we have formalized as follows:

$$
\exists j, R_{j}^{A} \subseteq A^{n}, R_{j}^{B} \subseteq B^{n}, \exists \bar{a} \in A^{n}: R_{j}^{A}(\bar{a}) \wedge \neg R_{j}^{B}(f(\bar{a}))
$$

Let's start with homomorphism. The formula (1) describing the completeness condition is logically equivalent to the following formula: $\neg R_{j}^{A}(\bar{a}) \vee R_{j}^{B}(f(\bar{a}))$ whose logical contradiction $\neg\left(\neg R_{j}^{A}(\bar{a}) \vee R_{j}^{B}(f(\bar{a}))\right)$ is, in turn, equivalent to the formula for abstracting $R_{j}^{A}(\bar{a}) \wedge \neg R_{j}^{B}(f(\bar{a}))$. In other words, the condition of completeness is logically incompatible with abstraction. Yet, epimorphism and isomorphism, both in their standard version and in the version where only faithfulness is weakened, all satisfy completeness. Therefore epimorphism, epimorphism ${ }^{f}$, isomorphism and isomorphism ${ }^{f}$ are logically unsuited to accommodate abstraction.

The second form of misrepresentation is pretence, which we have formalized as follows:

$$
\exists j, R_{j}^{A} \subseteq A^{n}, R_{j}^{B} \subseteq B^{n}, \quad \exists \bar{b} \in B^{n}, \bar{a} \in A^{n}, \bar{b}=f(\bar{a}): \neg R_{j}^{A}(\bar{a}) \wedge R_{j}^{B}(f(\bar{a}))
$$


We have just seen that homomorphism and, more precisely, the condition of completeness, is logically equivalent to the formula: $\neg R_{j}^{A}(\bar{a}) \vee R_{j}^{B}(f(\bar{a}))$. Hence, homomorphism allows for pretence as a logical possibility. On the other hand, pretence logically contradicts faithfulness. Indeed, the formula (2) for faithfulness is equivalent to $R_{j}^{A}(\bar{a}) \vee \neg R_{j}^{B}(f(\bar{a}))$ whose logical contradiction is exactly $\neg R_{j}^{A}(\bar{a}) \wedge R_{j}^{B}(f(\bar{a}))$. Therefore, any morphism that satisfies faithfulness can not accommodate pretence. This evidently holds for epimorphism and isomorphism. What about the weakened version of faithfulness? We have seen that weakening faithfulness admits of a tuple $\bar{a} \in A$ which does not stand in relation $R_{j}^{A} \subseteq A^{n}$ even though its image $\bar{b} \in B$ stands in the the corresponding relation $R_{j}^{B} \subseteq B^{n}$. Weakened faithfulness, then, allows pretence in principle. However, for weakened faithfulness to actually accommodate pretence, it is crucial that the function $f$ is not injective, otherwise weakened faithfulness can not accommodate pretence. Therefore, epimorphism ${ }^{* f}$ accommodates pretence, but epimorphism, isomorphism and isomorphism ${ }^{* f}$ do not accommodate this form of misrepresentation.

The third form of misrepresentation is simulation, which we have formalized as follows:

$$
\begin{gathered}
\exists j, k, R_{j}^{A}, R_{k}^{A} \subseteq A^{n}, R_{j}^{B}, R_{k}^{B} \subseteq B^{n}, \exists \bar{a} \in A^{n}, \bar{b} \in B^{n}, \bar{b}=f(\bar{a}): \\
\underbrace{\left(R_{j}^{A}(\bar{a}) \wedge \neg R_{j}^{B}(f(\bar{a}))\right)}_{\text {abstracting on } \bar{a}, \bar{b}} \wedge \underbrace{\left(\neg R_{k}^{A}(\bar{a}) \wedge R_{k}^{B}(f(\bar{a}))\right)}_{\text {pretending on } \bar{a}, \bar{b}}
\end{gathered}
$$

Simulation is what obtains from both abstracting and pretending on the same tuple, which is a common phenomenon in modeling (as stressed by Cartwright 1989, Frigg and Hartmann 2006). In this case, it is much easier to verify which form of misrepresentation is accommodated by which kind of morphism, given that we just need to jointly consider what abstracting and pretending allow for. It is then the case that only homomorphism ${ }^{* c}$ and epimorphism ${ }^{* f, c}$ accommodate simulation, and neither of them are proper morphisms that can transfer structure.

In table (2) we summarize the results of our analysis, which leads us to conclude that no morphism that can be said to transfer structure from a source to a target is actually able to accommodate all forms of inaccurate misrepresentation. The structural mappings that merely satisfy weakened versions of completeness can not be said to transfer structure, and the rest are unable to accommodate at least one main form of misrepresentation as inaccuracy. Therefore we conclude that isomorphism, epimorphism and homomorphism all fail to account for the phenomenon of misrepresentation.

It is in particular startling that most of the structural accounts proposed so far fail to accommodate the one form of misrepresentation as abstraction that philosophers of science have entertained ever since the times of Cambpell's influential discussion of the kinetic theory of gases. While structural mappings can be very helpful in establishing the accuracy of certain mathematical representations in physics, they are unable to characterize the very relation of representation in general. 


\begin{tabular}{c|c|c|c|} 
& Abstraction & Pretence & Simulation \\
\hline Homomorphism & NO & YES & NO \\
\hline Epimorphism & NO & NO & NO \\
\hline Epimorphism $^{* f}$ & NO & YES & NO \\
\hline Isomorphism $^{*}$ & NO & NO & NO \\
\hline Isomorphism $^{* f}$ & YES & NO & NO \\
\hline
\end{tabular}

Table 2: Morphisms and Inaccuracy

\section{Conclusions}

We have examined Bartels' homomorphism theory of scientific representation. We have examined it in relation to two typical kinds of misrepresentation in scientific models, which we may refer to as 'mistargetting' and 'inaccuracy'. The former involves choosing the wrong target for a modeling source on account of perceived similarities or structural matches, and shows representation to be an essentially intentional notion (in a broad sense that encompasses intended use). The latter involves at least three different kinds of distortion of model targets by model sources, which we have distinguished as abstraction, pretence and simulation. We have illustrated these distinctions by means of a careful study of the historical case of the billiard ball model. This model was notoriously invoked by Mary Hesse in her rightly influential work on analogy. Nevertheless Hesse's treatment of the model is itself highly idealized. We claim that there is more to the actual case study than just positive and negative analogies in the sense discussed by Hesse. In particular there are inverse negative analogies, or analogies 'by denial', as well as negative analogies by 'abstraction': there are properties of gas molecules that billiard balls lack, as well as properties of billiard balls that gas molecules lack. We then endeavored to provide formal characterizations for all these distinctions in a form that is suitable to the homomorphism account of representation. The taxonomy thus obtained proves useful to determine whether homomorphism - or indeed any other kind of morphism - accommodates misrepresentation.

We share with Bartels the thought that the adequacy of any account of scientific representation demands such accommodation. Any adequate account must at least accommodate, if not explain, mistargetting and the three kinds of inaccuracy we have discussed. Now, as for mistargetting, we have examined whether Bartels' account successfully cope with it. A closer analysis has revealed some issues remain regarding how much work effectively homomorphism is doing in the account. We have argued that the representational mechanism that Bartels appeals to is crucial in determining representation,misrepresentation or nonrepresentation. Thus, there does not seem to be much work left for homomorphism to do. Bartels does claim that homomorphism is necessary for representation or misrepresentation alike, yet his actual discussion of the role played by the representational mechanism seems prima facie to belie this claim. As for the three forms of inaccuracy that we have discussed, we have provided arguments to the effect that while homomorphism may account for pre- 
tence - although not in the form of epimorphism actually defended by Bartels - it can not provide for abstraction. We thus concluded that, contrary to Bartels' claim, the homomorphism account can not provide for any of the two typical kinds of misrepresentation by scientific models.

A structural account may well be needed to assess the accuracy or faithfulness of a scientific model, particularly in those cases where the model source and target can both be given appropriate structural descriptions. Nonetheless, even in such cases, it does not seem to be the case that the representational relation, or activity, is constituted by any structural morphism. It is rather what Bartels refers to as the "representational mechanism" that does all the conceptually required work at this stage. Once this basic mechanism is in place, it becomes appropriate to ask questions regarding the structural match of sources and targets. Representation does not essentially consist in transfer of structure from target system to source object. And while the homomorphism account may describe the means whereby some mathematical representations operate in science, it can not fully describe representation per se.

\section{Acknowledgements}

We would like to thank two anonymous referees for helpful comments and suggestions. Mauricio Suárez acknowledges the support of the European Commission (Marie Curie grant FP7-PEOPLE2012-IEF: project grant 329430) and of the Spanish Government (DGICT grant: FFI2014-57064P), and helpful discussions at the Universities of London, Florence, and Complutense of Madrid. Francesca Pero acknowledges the support of the Department of Philosophy, University of Florence and of the Complutense University of Madrid, as well as the helpful exchanges at the SILFS conference held in Rome, June 2014, and at the Universities of Florence and Complutense of Madrid. 


\section{References}

Bartels, A. (2006). Defending the structural concept of representation. Theoria, 55:7-19.

Borges, J. L. (1954). Del rigor en la ciencia. In Dutton, E. P., editor, Historia Universal de la Infamia. Emece, Buenos Aires. Translated as "On Exactitude in Science", in A Universal History of Infamy, E. P. Dutton, 1972.

Callender, C. and Cohen, J. (2006). There is no special problem about scientific representation. Theoria, 55:67-85.

Campbell, N. R. (1957). Foundations of Science: The Philosophy of Theory and Experiment. Dover Publication, New York.

Cartwright, N. (1983). How the Laws of Physics Lie. Oxford University Press, Oxford.

Cartwright, N. (1989). Nature's Capacities and their Measurement. Clarendon Press, Oxford.

Chakravartty, A. (2010). Informational versus functional theories of scientific representation. Synthese, 172:197-213.

Chang, C. C. and Keisler, H. J. (1973). Model Theory. Elsevier, Amsterdam.

Contessa, G. (2007). Scientific representation, interpretation, and surrogative reasoning. Philosophy of Science, 74:48-68.

Contessa, G. (2011). Scientific models and representation. In French, S. and Saatsi, J., editors, The Continuum companion to the Philosophy of Science, pages 120-137. Continuum, London.

Dunn, M. and Hardegree, G. (2001). Algebraic Methods in Philosophical Logic. Clarendon Press, Oxford.

Frigg, R. (2006). Scientific representation and the semantic view of theories. Theoria, 55:49-65.

Frigg, R. and Hartmann, S. (2006). Scientific models. In Sarkar, S. and Pfeifer, J., editors, The Philosophy of Science: An Encyclopedia, pages 740-749. Routledge, New York.

Giere, R. N. (1988). Explaining Science: A Cognitive Approach. University of Chicago Press, Chicago.

Giere, R. N. (1999). Using models to represent reality. In Magnani, L., Nersessian, N., and Thagard, P., editors, Model-based reasoning in scientific discovery, pages 41-57. Plenum, New York.

Hesse, M. (1970). Models and Analogies in Science. Notre Dame University Press, Notre Dame.

Hodges, W. (1997). A Shorter Model Theory. Cambridge University Press, New York.

Hodges, W. and Scanlon, T. (2013). First-order model theory. In Zalta, E. N., editor, The Stanford Encyclopedia of Philosophy. http://plato.stanford.edu/archives/fall2013/entries/modeltheory-fo/, fall 2013 edition.

Pincock, C. (2011). Philosophy of mathematics. In French, S. and Saatsi, J., editors, The Continuum Companion to the Philosophy of Science, pages 314-336. Continuum, London.

Pincock, C. (2012). Mathematics and scientific representation. Oxford University Press, Oxford.

Robinson, A. (1963). Introduction to model theory and to the metamathematics of algebra. North-Holland, Amsterdam.

Rothmaler, P. (2005). Elementary epimorphisms. Journal of Symbolic Logic, 70:473-487. 
Suárez, M. (2003). Scientific representation: against similarity and isomorphism. International studies in the philosophy of science, 17:226-244.

Suárez, M. (2004). An inferential conception of scientific representation. Philosophy of Science, 71:767-779.

Suárez, M. (2010). Scientific representation. Philosophy Compass, 5:91-101.

Suppes, P. (1967). What is a scientific theory? In Morgenbesser, S., editor, Philosophy of Science Today, pages 55-67. Basic books, New York.

Teller, P. (2001). Twilight of the perfect model model. Erkenntnis, 55:393-415.

Teller, P. (2008). Representation in science. In Psillos, S. and Curd, M., editors, The Routledge Companion to the Philosophy of Science, pages 435-441. Routledge, London.

van Fraassen, B. C. (1980). The Scientific Image. Oxford University Press, New York.

van Fraassen, B. C. (2006). Structure: Its shadow and substance. British Journal for the Philosophy of Science, 57:275-307.

van Fraassen, B. C. (2008). Scientific Representation: Paradoxes of Perspective. Oxford University Press, New York. 